\title{
Gender Classification of Complex Face Images Based on AdaBoost
}

\author{
Junrui Wang, Qichuan Tian, Manli Wang, Xiaohui Wu \\ Beijing University of Civil Engineering and Architecture, Beijing 100044 \\ 1187436069@qq.com
}

Keywords: machine learning, gender classification, Adaboost, feature extraction

\begin{abstract}
This paper proposes a face-based gender classifier which is based on Adaboost, and selects the face database with different color, angle and illumination. A variety of feature extraction methods are used to reduce the dimension, noise and calculation of the sample, and ensure a high recognition rate. The simulation results show that the proposed classifier can complete the gender classification work with the interference of skin color, angle and illumination, and the error rate is only $7.5 \%$, what is more, the training and recognition speed is also get improved.
\end{abstract}

\section{Introduction}

Face recognition is an important part of biometric identification technology and one of the most challenging research topics. The face image contains rich features, including gender, age, race, identity, etc., among them, the gender classification based on face image is of great significance and has a wide application prospect. ${ }^{[1]}$

The gender classification algorithm of face image mainly contains supported vector machine $(\mathrm{SVM})^{[2]}$, linear discriminant algorithm (LDA) ${ }^{[3]}$ and AdaBoost (Adaptive Boosting) algorithm. Among these algorithm, the recognition rate of the SVM algorithm is relatively high, while its recognition speed is relatively slow. The LDA algorithm is more concerned with distinguishing the difference of face. AdaBoost algorithm is widely used in face recognition in recent years. The advantage of Adaboost lies in its high efficiency, just need to know the sample category, you can continue to learn through multi-layer training and obtain better recognition results. Therefore, this paper chooses AdaBoost algorithm to carry on the gender classification of face image.

AdaBoost algorithm is an important feature classification algorithm in machine learning, and has been widely used in applications such as face detection and image retrieval. In addition, this algorithm is often used for feature selection and feature weighting. There is a widely application of the AdaBoost algorithm.

The gender classification based on face image mainly includes three parts: face detection, feature extraction and classifier. In this paper, a variety of dimensionality reduction methods is used for facial image feature extraction and dimension reduction, and it also talks about training AdaBoost classifier to achieve gender classification of face images. The second section introduces the AdaBoost algorithm, the third section discusses the AdaBoost gender classification method adopted in this paper, the fourth section is simulation experiment, and the last section summarizes the whole paper.

\section{AdaBoost algorithm}

\subsection{The classification model of AdaBoost algorithm}

AdaBoost is an iterative algorithm whose core idea is to train different weak classifiers for the same training set and then integrate these weak classifiers to form a strong classifier. The algorithm itself is achieved by changing the data distribution, which determines the weight of each sample based on whether each sample classification in each training set is correct and the accuracy of the last overall classification ${ }^{[5]}$. The new data set with the modified weight is given to the lower classifier for training, and finally the classifier of each training is integrated to become the final 
strong classifier. The practical AdaBoost classifier can eliminate some unnecessary training data features and focus on training critical data.

\subsection{The process of the AdaBoost algorithm}

The error rate of the classifier tends to zero as long as each weak classifier classification capability in AdaBoost is better than random guessing, and when its number tends to infinity ${ }^{[6]}$. The structure of the AdaBoost algorithm is shown in the following figure:

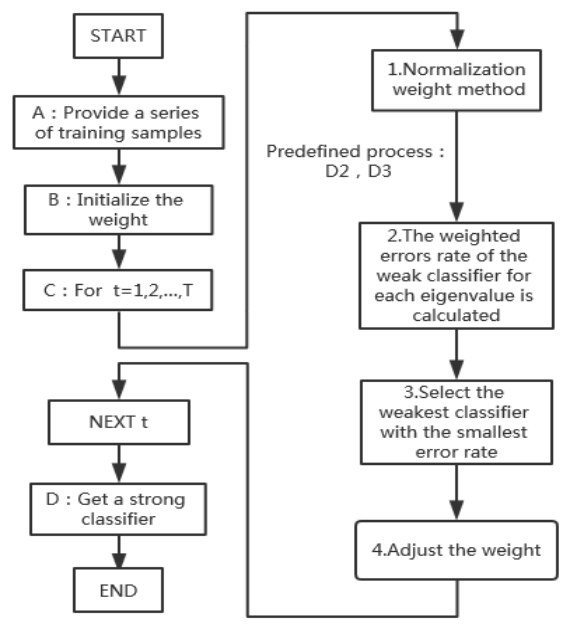

Figure 1 Adaboost algorithm flowchart.

\subsection{The basic theory of AdaBoost}

The AdaBoost algorithm, as an improved version of Boosting, is almost identical in efficiency to the traditional Boosting algorithm, but it does not require any prior knowledge about the weak learner, making it easier to apply it to practical problems ${ }^{[6]}$. The following is a formal description of the AdaBoost algorithm:

Given the training set: $\left(x_{1}, y_{1}\right), \ldots,\left(x_{N}, y_{N}\right)$, where $y_{i} \in\{-1,1\}$, that $\mathrm{x}_{\mathrm{i}}$ is the correct category label, $1, \ldots, N$. The initial distribution of samples on the training set is the following:

$$
D_{1}(i)=\frac{1}{N}
$$

For $\mathrm{t}=1 \ldots \mathrm{T}$, the weak classifier should be found with the smallest error rate on the distribution search: $\rightarrow\{-1,1\}$, where the error rate of a weak classifier is:

$$
\varepsilon_{\mathrm{t}}=\mathrm{P}_{\mathrm{D}_{\mathrm{t}}}\left(\mathrm{h}_{\mathrm{t}}\left(\mathrm{x}_{\mathrm{i}}\right) \neq \mathrm{y}_{\mathrm{i}}\right)
$$

Calculate the weighting factor for the weak classifier:

$$
\alpha_{\mathrm{t}}=\frac{1}{2} \ln \left(\frac{1-\varepsilon_{\mathrm{t}}}{\varepsilon_{\mathrm{t}}}\right)
$$

Update the distribution of training samples:

$$
D_{t+1}(i)=\frac{D_{t}(i) \exp \left(-\alpha_{t} y_{i} h_{t}\left(x_{i}\right)\right)}{Z_{t}}
$$

Where $\mathrm{Z}_{\mathrm{t}}$ is the Normalized constant.

The last strong classifier is:

The training error is analyzed as follows:

$$
\mathrm{H}_{\text {final }}(\mathrm{x})=\operatorname{sign}\left(\sum_{\mathrm{t}=1}^{\mathrm{T}} \alpha_{\mathrm{t}} \mathrm{h}_{\mathrm{t}}(\mathrm{x})\right)
$$


Set $\varepsilon_{t}=\frac{1}{2}-\gamma_{t}$, because the error rate of the weak classifier is less than the random guess, so $\gamma_{t}>0$, its training error is:

$$
\mathrm{R}_{\mathrm{tr}}\left(\mathrm{H}_{\text {final }}\right) \leq \exp \left(-2 \sum_{\mathrm{t}=1}^{\mathrm{T}} \gamma_{\mathrm{t}}^{2}\right)
$$

Set $\forall \mathrm{t}, \gamma \geq \gamma_{t}>0$, so we can get $R_{t r}\left(H_{\text {final }}\right) \leq e^{-2 r^{2} T}$, and we draw a conclusion that with the increase in the number of training rounds, the upper bound of training errors will continue to decrease.

\section{Gender classification based on AdaBoost}

\subsection{Face database and feature extraction}

There are some commonly used face database like FERET, ORL, Yale, PIE, AR, BioID, etc., this paper selected FERET face database. FERET face image database is initiated and established by the US Department of Defense to promote the face recognition algorithm further research and practical application ${ }^{[7]}$. From 180 people face images which is randomly selected by the FERET, including men and women of 90, 5 images for each person, covering a different perspective, expression, lighting conditions, and consist of different color of the face image . Randomly selected 140 groups of face images are uesd as a training group, men and women each 70; the remaining 40 groups as a test group, men and women each 20 .

The size of selected face image is $80 \times 80$, and it has been pre-processed. The face detection and segmentation process is not described in detail here. In this paper, MB-LBP and principal component analysis are used to extract the feature images of facial images, and the AdaBoost gender classifier is used to compare the test results. The training process and the comparison results are described in detail in the simulation experiment section.

\subsection{AdaBoost classifier training}

The Adaboost gender classifier's training process can be formally described as the following steps:

Given the set of male and female training samples, the sample set contains two matrices: male and female face image sample matrix, gender marker matrix. Male is marked as -1 , and female is marked as 1 .

The initial distribution of the training sample set is $1 / p$, which means that the weight of each training sample is $1 / \mathrm{p}$.

After the iteration of $\mathrm{T}$ rounds, a weak classifier is obtained after each iteration. The classifier is used to test the training sample and can obtain an error rate for the training sample, and then use the error rate to the sample value of the traversal update. If a training sample was wrong, then increase the weight of this sample, so that the next round of training will try to classify this wrong sample correctly. Then we follow the training set after modifying the weights, and we get the new weak classifiers h1, h2 ... ht.

Through this multi-round iteration and update the sample weight of the voting training,we ultimately get a strong classifier:

$$
H_{\text {final }}(x)=\operatorname{sign}\left(\sum_{t=1}^{T} \alpha_{t} h_{t}(x)\right)
$$

The best weak classifier obtained by each iteration. It is the weight obtained by the size of the classification error rate of the weak classifier got in each iteration. The better the classification effect is, the greater the weight is. Since the weight of each sample is introduced, each round of training is a process of deepening learning, so the more the number of iterations, the more knowledge the strong classifier has, and the better the classification performance will be. 


\section{Simulation experiment}

Input the male and female training group images (each $70 * 5$ ), a total of 700 and generate a 700 * 6400 training sample matrix: faces_ori. At the same time, generating a corresponding $700 * 1$ gender matrix faceslabel; input men and women each $20 * 5$ total 200 test image and then generate a $200 * 6400$ test sample matrix: faces_oritest, and the corresponding gender marker matrix facestlabel. In the gender marker matrix, the male is -1 and the female is 1 .

\subsection{Determine the optimal number of iterations for the classifier}

Take training samples and test samples to a simple resampling process in order to treduce their dimensions to $700 * 800$ and $200 * 800$ as training and test samples for this section. After selecting the number of iterations every 10 in the range of $[10,700]$, training the gender classifier, observeing the training and the test error rate, we can get the optimal number of iterations of the classifier. The results are shown in the following figure:
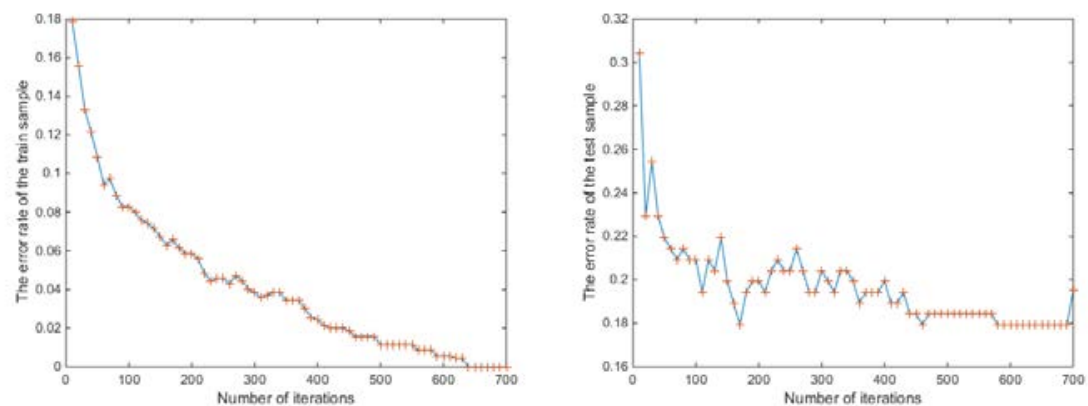

Figure 2 Error Rate Chart of Adaboost Classifier with Different Iterations.

As it can be seen from the above figure, when the number of iterations reaches 630 , the training error of the classifier is reduced to zero and the test error is $17.9 \%$ after 590 times and basically stabilizes. Thus, the optimal number of iterations of the classifier can be chosen 630 times. As the face image selected in this paper contains different interference factors: races, angles, lighting environment, so the required number of iterations is relatively high.

\subsection{Feature extraction and classifier training}

Simple resampling, PCA feature extraction and MB-LBP feature extraction were used to train the gender classifier, and the training effect of PCA method and MB-LBP operator with different dimensions were analyzed. In addition, the experimental results of the three methods were analyzed and compared.

The original image is $80 \times 80$ face image, the input is 6400-dimensional data, after a simple resampling processing, it will reduce the dimension to a certain value. In the range of $[50,1000]$ every 50 select a target dimension, training classifier and get the test results, the following figure is a simple resampling processing classifier training results:

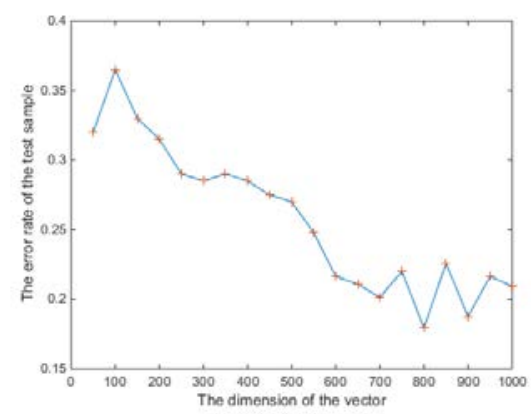

Figure 3 Error Rate Chart of Adaboost Classifier with changing vector.

In order to obtain the best dimensionality reduction, we use the method of fast PCA to reduce the dimension and choose a target descending dimension every 10 in the range of $[10,200]$, and train the classifier separately, as shown in the figure below. The experimental results show that, the 
classifier, which is trained by the reduced dimension PCA, The overall error rate varies dramatically and the error rate is more than $25 \%$. Therefore, when the principal component is used as a feature, the training of gender classifier performance is poor.

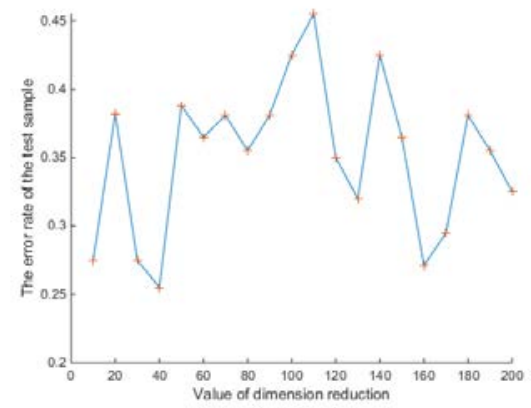

Figure 4 The Error Rate Chart of PCA test sample.

By comparing the effect of the three operators, the feature matrix generated by $\mathrm{MB}_{2}-$ $\mathrm{LBP}_{8,2}^{\mathrm{u}_{2}}$ operator is chosen as the characteristic sample. The dimension of each sample vector is 1296 and the number of iterations is 640. And then in the [0 1000] interval every 50 select a dimension, and the sample vector down to this dimension, and then after the reduction of training samples and test samples to test, and get the wrong sample test rate, the following is the experiment results:

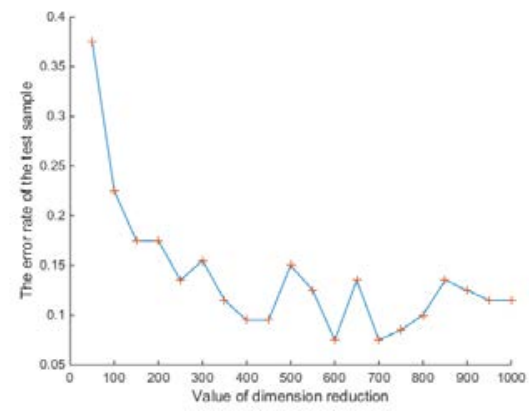

Figure 5 The Error Rate Chart of LBP test sample.

It can be seen from the experimental results that the classifier trained by the feature matrix generated by the $\mathrm{MMB}_{2}-\mathrm{LBP}_{8,2}^{\mathrm{u}_{2}}$ operator is classified as a gender classifier with an error rate of $7.5 \%$ when the classifier is reduced to 600 dimension. The classifier has successfully removed the noise effect, achieved a better recognition effect, and successfully reduced the feature dimension to 600 dimensions, greatly reducing the amount of computation, thereby reducing the training and testing time. Compared to the previous classifier, this classifier performance is excellent.

\section{Conclusion}

In this paper, a face database with different color, angle and illumination is selected, and the face gender classifier based on Adaboost with different feature extraction methods is trained, and the training results are compared and analyzed. The simulation results show that the gender classifier based on Adaboost can complete the gender classification under the interference of skin color, angle and illumination, but it still needs to reduce the error rate by optimizing the weak classifier and iterative method. In this paper, the best feature extraction method is MB-LBP, which successfully reduces noise and the computational cost and achieves the best classification effect. The work of this paper is still has some shortcomings, face database is not rich, the training database is still in a small scale. In the future, the research will focus on the face detection and cutting, and how to integrate the weak classifier into a strong classifier and so on.

\section{Acknowledgements}

This study is sponsored by the BUCEA Post Graduate Innovation Project. The General Program 
of Science and Technology Development Project of Beijing Municipal Education Commission under Grant No.KM201410016016, Beijing University of Civil Engineering and Architecture Science Research Foundation under Grant No.00331614021, and the Practical Training Plan of Beijing College High Level Talents Cross Developing Project support this work.

\section{References}

[1]Wu Bo, Ai Haizhou, Xiao Xipan, Xu Gauangyou.(2003) Gender classification of face image. Computer Research and Development, 1546-1553.

[2]Moghaddaxn B, Yang M H.(2002) Learning gender with support faces, Pattern Analysis and Machine Intelligence. IEEE Transactions on, 707-711.

[3]Buchala S, Davey N, Frank R, Gale T, Loomes M, Kanargard W.(2004) Gender classification of faces images: The role of global and feature-based information, ICONIP 3316.

[4]Jian-Gang Wang, Jun Li,and Wei-Yun Yau. (2010) Boosting Dense SIFT Descriptors and Shape Contexts of Face Images for Gender Recognition. IEEE Computer Society Conference on Computer Vision and Pattern Recognition Workshops (CVPRW), 96-102.

[5]R.E. Schapire, Y. Freund, P. Bartlett etc.,(1997) Boosting the Margin: A New Explanation for the Effectiveness of Voting Methods, Proc. Fourteenth International Conference on Machine Learning.

[6]LienhartR, MaydtJ.(2002)An Extended Set of Harr-like Features for Rapid Object Detection.IEEE ICIP , 900-903.

[7]Phillips P J, Wechsler H, Huang J, et al.(1998) The FERET database and evaluation procedure for face-recognition algorithms. Image and vision computing, 295-306. 